\title{
Fulminant myocarditis complicated with cardiac arrest and subsequent acute respiratory distress syndrome formation rescued by Extracorporeal Membrane Oxygenation - A rare case report
}

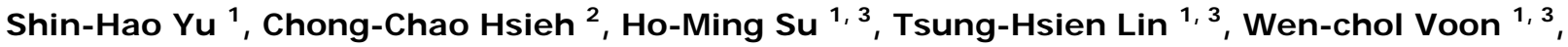 \\ Wen-Ter Lai ${ }^{1,3}$, Sheng-Hsiung Sheu ${ }^{1,3}$, Po-Chao Hsu 1, 4
}

1. Division of Cardiology, Department of Internal Medicine, Kaohsiung Medical University Hospital, Kaohsiung, Taiwan. 2. Division of Cardiovascular Surgery, Kaohsiung Medical University Hospital, Kaohsiung, Taiwan. 3. Department of Internal Medicine, Faculty of Medicine, Kaohsiung, Taiwan. 4. Graduate Institute of Medicine, College of Medicine, Kaohsiung Medical University, Kaohsiung, Taiwan.

Correspondence: Po-Chao Hsu. Address: Division of Cardiology, Department of Internal Medicine; Kaohsiung Medical University Hospital, 100 Tzyou 1st Road, Kaohsiung, 80708, Taiwan. Email: pochao.hsu@gmail.com

Received: February 16, $2014 \quad$ Accepted: March 28, $2014 \quad$ Online Published: May 12, 2014

DOI : 10.5430/crim.v1n2p116 URL: http://dx.doi.org/10.5430/crim.v1n2p116

\section{Abstract}

Acute fulminant myocarditis (AFM) is a relatively rare diagnosis characterized by heart failure preceded by symptoms of viral infection. Sometimes circulatory mechanical support is required for this catastrophic condition and mortality rate was still high. Adult respiratory distress syndrome (ARDS) was a rare complication of myocarditis in the literature. However, there was limited literature discussing AFM complicated with cardiac arrest and subsequent ARDS formation and the prognosis of extracorporeal membrane oxygenation (ECMO) treatment is unknown. Here we report a case of AFM suffering from cardiac arrest and ARDS formation and successfully treated by ECMO. This rare case reminds physicians that ARDS is still a possible but extremely rare complication of fulminant myocarditis, and ECMO is a lifesaving treatment not only for AFM with profound shock but also for possible ARDS formation.

\section{Keywords}

Extracorporeal membrane oxygenation, Fulminant myocarditis, Adult respiratory distress syndrome

\section{Introduction}

Acute fulminant myocarditis (AFM) is a relatively rare diagnosis characterized by heart failure preceded by symptoms of viral infection. It is a life-threatening medical emergency and the patients usually die because of ventricular arrhythmias and fulminant heart failure without immediate and adequate treatment. Circulatory mechanical support such as extracorporeal membrane oxygenation (ECMO) was reported to be a useful and life-saving strategy for AFM ${ }^{[1,2]}$. However, there was limited literature discussing AFM complicated with cardiac arrest and subsequent adult respiratory distress syndrome (ARDS) formation and the prognosis of ECMO treatment is unknown. We report a case of AFM suffering from cardiac arrest and ARDS formation and successfully treated by ECMO. 


\section{Case presentation}

A 57-year-old female suffered from progressive dyspnea and cold sweating was brought to our emergent department (ED). Tracing back her history, patient had history of hypertension with valsartan 160mg daily use, but denied any other systemic disease before. She had an episode of common cold for days before ED visit, with intermittent fever, nausea and vomiting. However, she did not pay attention to the symptoms. On ED presentation, blood pressure was 110/78 mmHg, heart rate 127 beats per minute, respiratory rate 16 breaths per minute, and body temperature $37.8^{\circ} \mathrm{C}$. Abdominal computer tomography was performed to rule out the possibility of intra-abdominal infection; bilateral pleural effusion, congestive liver, and infiltration over right middle and lower lung were found. Progressive dyspnea and hypotension were reported after the examination of abdominal computer tomography and high-dose vasopressors (dopamine $16.67 \mathrm{ug} / \mathrm{Kg} / \mathrm{min}$ and norepinephrine $10.67 \mu \mathrm{g} / \mathrm{min}$ ) were then given for shock status. First electrocardiogram (ECG) showed complete AV block, ST elevation over lead V3-V4 and pathologic Q wave over lead II, III, and aVF (see Figure 1A). However, follow-up ECG revealed axis change on precordial leads, first-degree AV block, and ST elevation over lead V1-V4 (see Figure 1B). In addition, laboratory data also revealed increased levels of cardiac biomarkers (Creatine kinase MB: 36.5

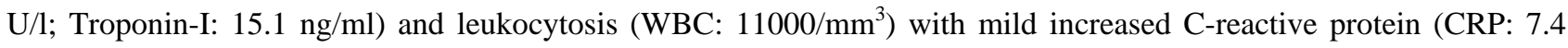
$\mathrm{mg} / \mathrm{L}$ ). Myocarditis was highly suspected according to the above findings but acute myocardial infarction (AMI) still could not be completely ruled out. Coronary angiography was arranged for further evaluation only showing left circumflex artery with 50\% stenosis. While patient was prepared for insertion of intra-aortic balloon pumping (IABP) sudden-onset cardiac arrest occurred. Emergent cardiopulmonary resuscitation (CPR) was started and endotracheal intubation was immediately performed. During CPR, temporary pacemaker was inserted for cardiac arrest and pulseless electrical activity (PEA), but it was still not effective. Due to persistent cardiac arrest without recover of spontaneous circulation, veno-arterial extracorporeal membrane oxygenation (ECMO) was started for life-saving and hemodynamic support. Then the patient was transferred to our cardiac care unit (CCU) for further intensive care.

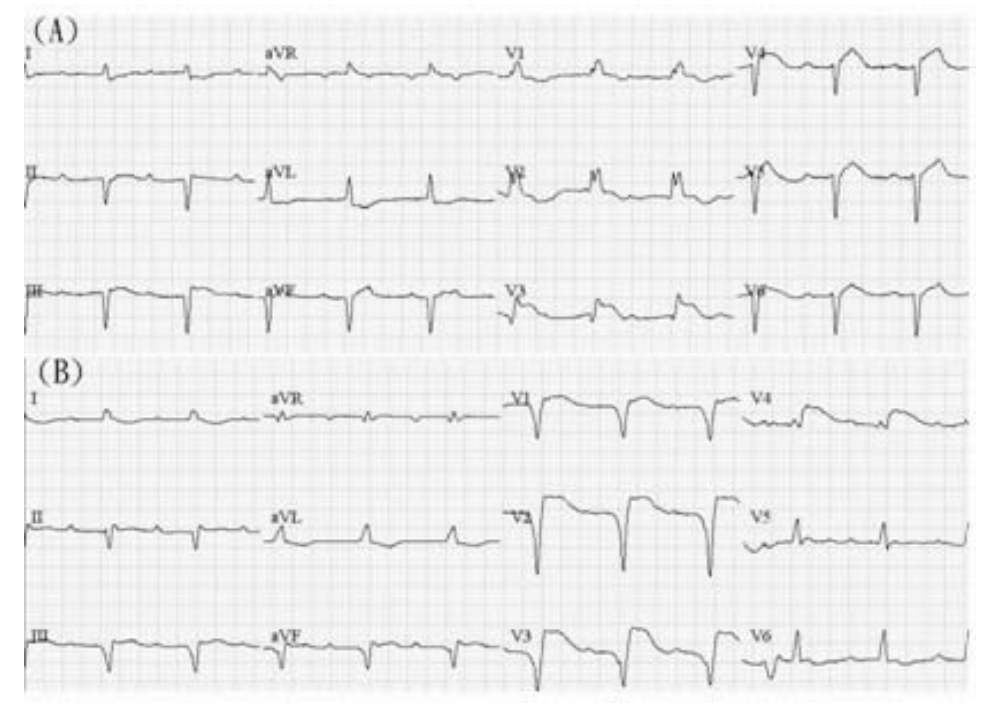

Figure 1A (Left panel) First electrocardiogram (ECG) showed complete AV block, ST elevation over lead V3-V4 and pathologic Q wave over lead II, III, and aVF. Figure 1B (Right panel) Follow-up ECG revealed axis change of precordial leads, first-degree AV block, and ST elevation over lead V1-V4.

On CCU bed rest, first echocardiographic evaluation revealed total left ventricle (LV) akinesia. Chest X-ray showed acute pulmonary edema with bilateral infiltration (see Figure 2A). Continuous venovenous hemodialysis was started for correction of metabolic acidosis and balance of body fluid status. Empirical antibiotics therapy with ceftazidime and vancomycin was started for potential infection later shifted to meropenem due to elevated CRP level (213.58mg/L). Follow-up on cardiac biomarkers showed peaked troponin I level was $19.954 \mathrm{ng} / \mathrm{ml}$. Both of dopamine and norepinephrine 
were gradually tapered off due to improved hemodynamics under IABP and ECMO support. Cardiac rhythm was gradually recovered to sinus rhythm but LV function evaluated by echocardiography still revealed nearly total akinesia in the first 5 days of CCU stay. Heart function gradually improved since the 6th day of CCU bed rest showing significant improvement in wall motion in next two days (LV ejection fraction: 59\%). Therefore, blood flow of ECMO was gradually decreased because of recovery of heart function. Urine amount of the patient was also significantly improved under intravenous furosemide use and mechanical support.

Unfortunately, defective oxygenation was noted subsequently and the FiO2 setting of ventilator was up-titrated from $60 \%$ to $100 \%$. CXR follow-up showed white-out of bilateral lung but the level of central venous pressure was only $14 \mathrm{cmH}_{2} \mathrm{O}$, lower than previous level. ARDS was highly suspected, and ventilator was further modified according to ARDS setting with low tidal volume and high positive end-expiratory pressure (PEEP). Oxygenation became more stable and we successfully removed ECMO on the 11th day of CCU bed rest. Intravenous methylprednisolone 80mg per day was also given seven days later after ARDS formation.

Despite of relative stable condition, the patient still had prolonged hospitalization due to poor weaning of ventilator, hospital acquired pneumonia complicated with empyema and twice pneumothorax episode (see Figure 2B). Finally, the patient was successfully discharged after infection under control and improvement of pneumothorax.
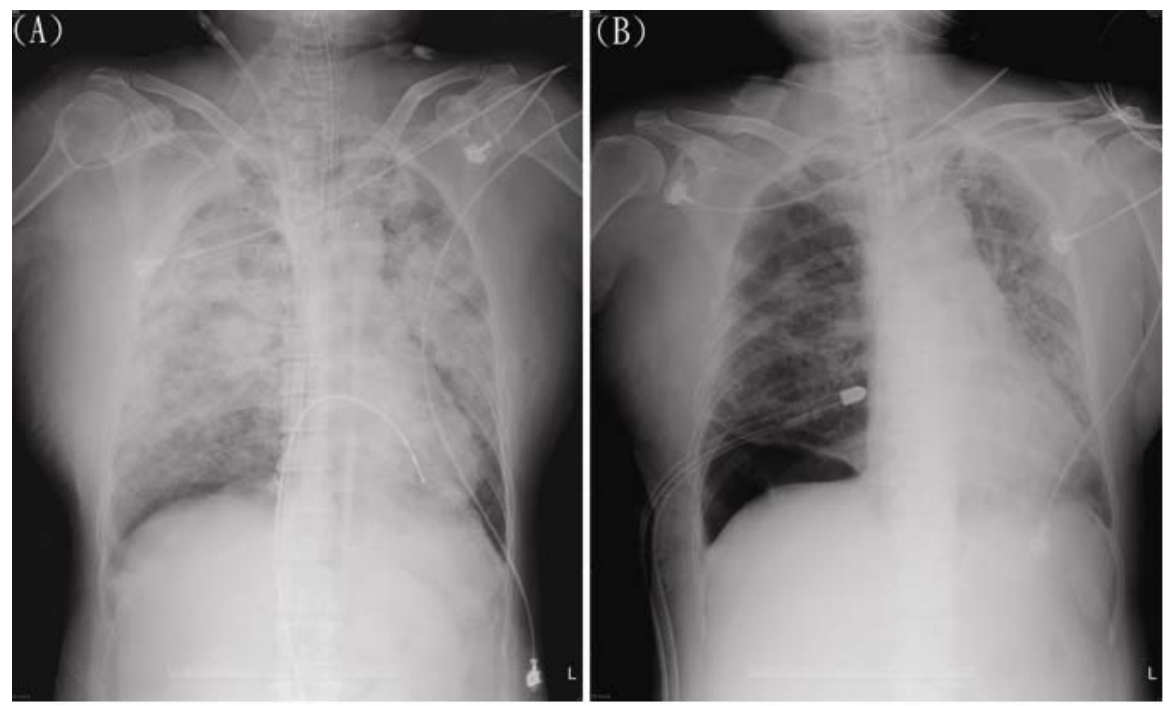

Figure 2A (Left panel) After admission to the cardiac care unit, chest X-ray (CXR) showed acute pulmonary edema with bilateral infiltration. Figure 2B (Right panel) CXR showed bilateral lung fibrotic change after acute respiratory distress syndrome episode and chest tube was inserted into right lung for treatment of pneumothorax.

\section{Discussion}

Myocarditis has a wide spectrum of clinical presentations, ranging from absence of clinical symptoms to fulminant cardiogenic shock. The electrocardiographic findings are often variable ${ }^{[3,4]}$. It can frequently mimic AMI ${ }^{[3,5,6]}$ and most of these cases were reported to have normal coronary arteries ${ }^{[5,6]}$ and long-term prognosis is generally good ${ }^{[3]}$. However, AFM is a life-threatening medical emergency and mortality was extremely high without immediate and adequate treatment ${ }^{[1]}$. Circulatory mechanical support such as ECMO was reported to be a useful and life-saving strategy for $\mathrm{AFM}^{[1,2]}$. Hsu KH et al. reported that ECMO can be a first-line tool to rescue the patients of AFM due to its simplicity and effectiveness. However, AFM still carries high mortality rates (36\%) in spite of advanced mechanical support ${ }^{[1]}$. Chung SY et al. also performed a single-center study to survey the outcome of patients with profound cardiogenic shock after CPR and prompt ECMO support. Although group of AFM had relative good prognostic outcome, it still had 53.8\% 
mortality in their study ${ }^{[2]}$. However, there is limited literature discussing AFM complicated with ARDS and the prognosis of ECMO treatment is still unknown. Although some pathogens such as dengue virus and scrub typhus were reported to be a possible cause which leaded to both of myocarditis and ARDS, the severity of myocarditis in these cases were not so high that circulatory mechanical support was not used ${ }^{[7,8]}$. In our case, AFM was highly suspected according to the following reasons: First, this patient had a history of virus-like infection several days previous to admission. Second, AMI was completely ruled out according to the result of coronary angiography. Third, echocardiography revealed total recovery of segmental wall motion and LV function from initial akinesia of LV. Fourth, serial ECG follow-up showed a variety of conduction disturbances before adequate treatment. Furthermore, our case suffered from prolonged cardiac arrest and pulseless electrical activity for five days under ventilator, IABP, and ECMO support which might suggest that the severity of fulminant myocarditis was extremely high. Although patient's heart function gradually improved under mechanical support, he suffered from another episode of ARDS formation which made the condition more complicated. ARDS is also a life-threatening condition of the respiratory system. Severe infection (pulmonary and extra-pulmonary) is one of the main etiologies of ARDS formation ${ }^{[9]}$. In addition to the setting of ventilator (low tidal volume and high PEEP), there are many different methods of ARDS treatment, including nitric oxide inhalation, prone position, surfactant therapy, and so on. However, ECMO represent last chance when all conventional treatments failed ${ }^{[10]}$. Therefore, ECMO use in our patient was not only useful for AFM, but also for ARDS formation.

Although patient survived from AFM complicated with cardiac arrest and ARDS formation, twice pneumothorax episode still occurred due to lung fibrosis and high PEEP-related barotrauma. Nevertheless, in this kind of high risk patients, prognosis of our case seemed not bad under ECMO support. Owing to limited case reports, further studies will be needed in the future.

In conclusion, this case report can remind physicians that ARDS is still a possible but extremely rare complication of fulminant myocarditis, and ECMO is a lifesaving treatment not only for AFM with profound shock but also for possible ARDS formation.

\section{References}

[1] Hsu KH, Chi NH, Yu HY, Wang CH, Huang SC, Wang SS, et al. Extracorporeal membranous oxygenation support for acute fulminant myocarditis: analysis of a single center's experience. Eur J Cardiothorac Surg. 2011 Sep; 40(3): 682-8.

[2] Chung SY, Sheu JJ, Lin YJ, Sun CK, Chang LT, Chen YL, et al. Outcome of Patients With Profound Cardiogenic Shock After Cardiopulmonary Resuscitation and Prompt Extracorporeal Membrane Oxygenation Support. Circ J. 2012 May 25; 76(6): 1385-92. http://dx.doi.org/10.1253/circj.CJ-11-1015

[3] Blauwet LA, Cooper LT. Myocarditis. Prog Cardiovasc Dis. 2010 Jan-Feb; 52(4): 274-88. http://dx.doi.org/10.1016/j.pcad.2009.11.006

[4] Nakashima H, Honda Y, Katayama T. Serial electrocardiographic findings in acute myocarditis. Intern Med. 1994 Nov; 33(11): 659-66. http://dx.doi.org/10.2169/internalmedicine.33.659

[5] Dec GW Jr, Waldman H, Southern J, Fallon JT, Hutter AM Jr, Palacios I. Viral myocarditis mimicking acute myocardial infarction. J Am Coll Cardiol. 1992 Jul; 20(1): 85-9. http://dx.doi.org/10.1016/0735-1097(92)90141-9

[6] Sarda L, Colin P, Boccara F, Daou D, Lebtahi R, Faraggi M, et al. Myocarditis in patients with clinical presentation of myocardial infarction and normal coronary angiograms. J Am Coll Cardiol. 2001 Mar 1; 37(3): 786-92. http://dx.doi.org/10.1016/S0735-1097(00)01201-8

[7] Deepak D, Garg R, Pawar M, Banerjee N, Solanki R, Maurya I. Filgrastim as a rescue therapy for persistent neutropenia in a case of dengue hemorrhagic Fever with acute respiratory distress syndrome and myocarditis. Case Rep Anesthesiol. $2011 ; 896783$.

[8] Sai Lakshmikanth Bharathi, S Jayachandran, N Senthil, S Sujatha. Scrub typhus causing myocarditis and ARDS: A case report. Heart India. 2013; 1: 85-6. http://dx.doi.org/10.4103/2321-449X.122785

[9] Mortelliti MP, Manning HL. Acute respiratory distress syndrome. Am Fam Physician. 2002; 65(9): 1823-1830.

[10] Ko WJ, Hsu HH, Tsai PR. Prolonged extracorporeal membrane oxygenation support for acute respiratory distress syndrome. J Formos Med Assoc. 2006 May; 105(5): 422-6. http://dx.doi.org/10.1016/S0929-6646(09)60140-6 